gegen einen Flächenstreik für unzulässig erklärt. Das ArbG Ulm unterstellt problemlos, daß erst die Aussperrung eine materielle Kampfparität herstellt, die Begründung für die Unzulässigkeit spezifischer Formen der Aussperrung erfolgt allein über den Begriff der "Verhältnismäßigkeit«.

[...] Bei Tarifvertragsverhandlungen sind die Arbeitnehmer und ihre Gewerkschaften notwendigerweise die Angreifer, während die Arbeitgeber den Forderungen der Gegenseite entgegensehen können. Der Kammer ist kein einziger Fall bekannt, in dem die Arbeitgeberseite genötigt wäre, die Angriffsaussperrung auszurufen. Es kann daher Art. 9 Abs. 3 Grundgesetz, der ja in erster Linie eine soziale Schutzgarantie enthält [...] und nur in diesem Zusammenhang einen Kernbereich des Tarifvertragssystems und der Arbeitskampffreiheit garantiert, nicht die Zulässigkeit der Angriffsaussperrung entnommen werden. Vielmehr ist die Angriffsaussperrung als Mißbrauch der Verfügungsmacht der Arbeitgeber über die Produktionsmittel rechtsunwirksam (Art. 20 Grundgesetz).

Das BAG ist auch inkonsequent, wenn es im Beschluß des Großen Senats vom 21. 4. I97 I (AP Nr. 43 zu Art. 9 Grundgesetz Arbeitskampf) einerseits postuliert, die Aussperrung habe in der Regel suspendierende Wirkung, könne im Rahmen der Verhältnismäßigkeit aber auch mit lösender Wirkung ausgerufen werden. Der Arbeitsplatz ist des Arbeitnehmers einzige Existenzgrundlage und der Ort, an dem er in erster Linie seine Persönlichkeit entfaltet. Dem Arbeitnehmer wegen eines vorübergehenden kollektiven Kampfzieles diesen Arbeitsplatz zu nehmen, ist nach der sicheren Überzeugung der Kammer in jedem Falle unverhältnismäßig; die lösende Aussperrung steht gerade in keinem vertretbaren Verhältnis zum vorübergehenden Kampfzweck, sie ist unsozial und daher aus Art. 9 Grundgesetz nicht ableitbar [...].

Für zulässig hält das Gericht aber eine suspendierende Aussperrung dann, wenn diese zur Ausübung von Gegendruck für den Abschluß ausgewogener Tarifverträge erforderlich ist. Insoweit wird die Aussperrung durch Art. 9 Abs. 3 Grundgesetz gedeckt. Danach ist eine suspendierende Abwehraussperrung im Falle eines Flächenstreiks unzulässig, weil nicht erforderlich und daher unverhältnismäßig. Gegen einen Schwerpunktstreik kann eine suspendierende Abwehraussperrung deshalb ausgrufen werden, weil ohne diesen Gegendruck die Gewerkschaften den Inhalt des Tarifvertrages ihrerseits diktieren könnten [. . .].«

Rainer Erd

\title{
Ein Grundrecht als Ausnahmerecht? \\ Anmerkung zum Kriegsdienstverweigerungs-Urteil des BVerfG
}

I.

Mit dem jüngsten Urteil des BVerfG zur Kriegsdienstverweigerung vom 1 3. 4. $1978^{\mathrm{I}}$ vollzog sich der vorletzte Schritt der verfassungsrichterlichen Uminterpretation eines ursprünglich vorbehaltlos garantierten Grundrechts zum Grundrecht mit numerus clausus. In Fortschreibung der Entscheidung aus dem Jahre I 970 zur Verpflichtung noch nicht anerkannter Kriegsdienstverweigerer zum Waffendienst ${ }^{2}$, mit der der "Funktionsfähigkeit der Bundeswehr" erstmals die Qualifikation zur Begrenzung des Grundrechts in einer einzelnen Beziehung verliehen worden war, wird nun, vermittelt über das Gleichheitsgebot des Art. 3 I GG, die Wehrpflicht zur generellen Verfassungsschranke in Art. 4 III GG erhoben. Die tragenden Entscheidungsgründe sind in den folgenden Leitsätzen zusammengefaßt: ${ }^{3}$

1 BVerfG NJW 1978,1245 ff. mit Sondervotum Hirsch.

2 BVerfGE 28, 243 (261); Zur Kritik dieser Entscheidung vgl. Kempen, Staatsraison über Verfassungsraison, JZ 1971, 452 ff.; Goerlich, Wertordnung und Grundgesetz, Baden-Baden 1973, S. 99 ff.; Krölls, Kriegsdienstverweigerung, Grundrecht zwischen Gewissensfreiheit und Kriminalität, Leverkusen 1977 , S. $23 \mathrm{Iff}$.

3 BVerfG NJW 1978, 1245. 
I. Die von der Verfassung geforderte militärische Landesverteidigung kann auf der Grundlage der allgemeinen Wehrpflicht, aber - sofern ihre Funktionstüchtigkeit gewährleistet bleibt - verfassungsrechtlich unbedenklich beispielsweise auch durch eine Freiwilligenarmee sichergestellt werden.

2. Die allgemeine Wehrpflicht ist Ausdruck des allgemeinen Gleichheitsgedankens. Ihre Durchführung steht unter der Herrschaft des Art. 3 I GG.

3. Kriegsdienstverweigerer aus Gewissensgründen sind gem. Art. I2a II i. V. mit Art. 4 III GG von Verfassungs wegen vom Wehrdienst nach Art. I 2a I GG befreit.

$[\ldots]$

5. Der Verfassungsgeber hat nicht eine allen Staatsbürgern - also gem. Art. 3 II GG auch dem weiblichen Teil der Bevölkerung - obliegende Dienstpflicht für das allgemeine Wohl zugelassen. Der in Art. 1 2a II GG vorgesehene Ersatzdienst ist vom Grundgesetz nicht als alternative Form der Erfüllung der Wehrpflicht gedacht; er ist nur Wehrpflichtigen vorbehalten, die den Dienst mit der Waffe aus Gewissensgründen verweigern.

6. Dem Verfassungsgebot der staatsbürgerlichen Pflichtengleichheit in Gestalt der Wehrgerechtigkeit wird nicht schon dadurch genügt, daß die Wehrpflichtigen entweder zum Wehrdienst oder zum Ersatzdienst herangezogen werden. Das Grundgesetz verlangt vielmehr, daß der Wehrpflichtige grundsätzlich Wehrdienst leistet, und verbietet es deshalb, in den als Ersatz des Wehrdienstes eingerichteten Zivildienst andere als solche Wehrpflichtige einzuberufen, die nach Art. r 2a II i. V. mit Art. 4 III GG den Dienst mit der Waffe aus Gewissensgründen verweigern dürfen.

7. Die Wehrgerechtigkeit fordert von jeder gesetzlichen Regelung nach Art. I 2a II GG i. V. mit Art. 4 III 2 GG, daß nur solche Wehrpflichtige als Kriegsdienstverweigerer anerkannt werden, bei denen mit hinreichender Sicherheit angenommen werden kann, daß in ihrer Person die Voraussetzungen des Art. 4 III I GG erfüllt sind. $\$ 25$ a I WpflG n. F. genügt diesem Erfordernis nicht.

8. Wie eine gesetzliche Regelung, welche die Ausgestaltung des Ersatzdienstes als einzige Probe auf die Gewissensentscheidung einsetzt, beschaffen sein muß, wenn sie der Verfassung entsprechen soll, hängt von zahlreichen Faktoren ab. Der Gesetzgeber hat insoweit innerhalb des von Art. I 2a II 2, 3 GG gezogenen Rahmens volle Gestaltungsfreiheit. Außer der Pflicht, Waffendienst zu leisten, kann er alle Pflichten und Belastungen, welche die Wehrdienstleistenden treffen, in gleichem Maße auch den Zivildienstleistenden auferlegen.

9. Angesichts des Mißverhältnisses zwischen der Zahl der verfügbaren Ersatzdienstpflichtigen und der Zahl der vorhandenen und besetzbaren Einsatzplätze im Zivildienst sowie im Hinblick darauf, daß der Gesetzgeber den ihm von Art. I 2a II 2, 3 GG für die rechtliche Ausgestaltung des Zivildienstes gezogenen Rahmen bislang nicht ausgeschöpft hat, kann die Ersatzdienstpflicht gegenwärtig nicht als eine im Verhältnis zur Wehrdienstpflicht auch nur gleichermaßen aktuelle und gleichbelastende Pflicht angesehen werden. [...]

II.

Die Argumentationsführung des Gerichts ist dadurch charakterisiert, daß die mit dem I. Leitsatz benannte Entscheidungsgrundlage eines angeblichen Verfassungsauftrags zur militärischen Landesverteidigung im folgenden nur noch in der verhüllten Form der Wehrgerechtigkeit auftritt ${ }^{4}$, so daß nicht die Funktionstüchtigkeit der Streitkräfte sondern der »unpolitische « Gleichheitssatz als Reformgrenze erscheint. Diese Konstruktion vermag freilich den Mangel ihres dogmatischen Ausgangspunktes nur oberflächlich zu kompensieren. Wortlaut, ratio legis, systematische Stellung und Entstehungsgeschichte der wehrrechtlichen Normen belegen, daß sich aus dem Grundgesetz ein Verfassungs-Befebl zur Unterhaltung von Streitkräften nicht herleiten läßt, sondern die Verfassung lediglich die Möglichkeit zu einer militärischen Landesverteidigung eröffnet. ${ }^{4^{a}}$ Während Art. I 2a I GG als Ausnahme von der in

\footnotetext{
4 Zur Kritik an der Einführung des «publizistischen Begriffes « der "Wehrgerechtigkeit " als grundrechtsdogmatischer Größe vgl. J. Ipsen, Wehrdienst, Ersatzdienst und Pflichtengleichheit, ZRP 1978, s 53 (ISS).

4a Vgl. insbesondere die grundlegende Untersuchung von Lutz-Rittberger, Abrüstungspolitik und Grund-
} 
Art. 12 GG garantierten Berufsfreiheit die Befugnis zur Einführung der allgemeinen Wehrpflicht erteilt, weist Art. 73 Nr. I GG dem Bund die ausschließliche Gesetzgebungs-Kompetenz für das Verteidigungswesen zu, die ihre Ergänzung in der Exekutiv-Kompetenz zur Aufstellung von Streitkräften durch Art. 87a I S. 1 GG findet.' Die Existenz der Bundeswehr steht somit jederzeit zur politischen Disposition des einfachen Bundesgesetzgebers. Der "verfassungsrechtliche VerteidigungsAuftrag « löst sich auf in ein von der Verfassung erteiltes politisches Mandat auf Widerruf: Wenn und solange die politischen Instanzen von der Ermächtigung zur Unterhaltung einer Armee Gebrauch machen, ist deren Funktion ausschließlich die der Abwehr oder Abschreckung eines äußeren Feindes.

Mangels einer Grundlage im Verfassungstext muß daher das BVerfG zu der apodiktischen Behauptung Zuflucht nehmen, »das Grundgesetz geh(e) ... davon aus, daß eine funktionsfähige, militärische Landesverteidigung aufgebaut " ${ }^{6}$ werde, die ihre Scheinlegitimation aus solch nebulösen Begrifflichkeiten wie einem »Verfassungsgrundsatz " bzw. einer "verfassungsrechtlichen Grundentscheidung für die militärische Landesverteidigung « $^{7}$ bezieht.

Die nächste Argumentationsstufe ist schon vorgezeichnet. Hat man einmal die militärische Landesverteidigung in den Rang eines überragenden Verfassungsgutes erhoben, so nimmt die allgemeine Wehrpflicht als eine der dem gesetzgeberischen Ermessen überantworteten Varianten zur Erfüllung des angeblichen Verfassungsauftrages an dieser Beförderung teil. Aus einer einfach-gesetzlichen Rechtspflicht wird eine »verfassungsrechtlich verankerte Pflicht $\aleph^{8}$ (als müsse nicht letztlich jede den Bürger gegenüber dem Staat treffende Verpflichtung ihre Grundlage in der Verfassung finden), die sich mit dem Gleichheitssatz zum »Verfassungsgebot der staatsbürgerlichen Pflichtengleichheit in Gestalt der Wehrgerechtigkeit « verbindet. Uber diese Figur der allumfassenden Pflichtengleichheit vollzieht sich dann die Unterordnung des Grundrechtes der Kriegsdienstverweigerung unter den Primat des Militärischen. Unter interessierter Abstraktion vom Stellenwert des Art. 4 III GG, der der »Pflicht, sich an der bewaffneten Landesverteidigung . . . zu beteiligen, eine unüberwindliche Schranke $\ll^{9}$ entgegensetzt, wird das Grundrecht der Kriegsdienstverweigerung zu einer speziellen Wehrdienst-Ausnahme degradiert. Der Personenkreis der Grundrechtsträger aus Art. 4 III GG, die von Verfassungs wegen gar nicht unter die allgemeine Wehrpflicht fallen, wird mit Wehrpflichtigen gleichgestellt, die wegen gesetzlicher Freistellungs- oder Zurückstellungsgründe von der Ableistung des Wehrdienstes ausgenommen sind.

Mit der Klassifizierung als Ausnahmerecht vollendet sich eine Entwicklung, die 1956 mit der Präsentation eines Regierungsentwurfs zum Wehrpflichtgesetz ${ }^{10}$ ihren

gesetz, Baden-Baden 1976, S. 37-81; ferner Menzel, BK, Nachtr. z. Art. 73 Nr. 1, Erl. II 2; K. Ipsen, BK, Art. 87a, Rdnr. 16-17; Hernekamp in v. Münch, Grundgesetz-Kommentar, Bd. 3, Art. 87, Rdnr. 8. - Anderer Ansicht: Wissenschaftlicher Dienst des Deutschen Bundestages (Fachbereich III Recht und Inneres), Gutachten zum Thema „Gibt es einen Auftrag des Grundgesetzes, die äußere Sicherheit zu gewährleisten? «, Bonn 1973; Hahnenfeld, Kriegsdienstverweigerung und der Verfassungsauftrag der Streitkräfte, NZWehrR 1975, I6ı ff.; Greve, Zur Frage der Verfassungsmäßigkeit eines Verzichts auf den Nachweis des Kriegsdienstverweigerungsrechts, BWV 1975, 64 ff.; BVerwG NJW 1976, 71 f. 2033 (2035), unter Berufung auf das obiter dictum in BVerfGE 28, 36 (47).

5 Dürig in: Maunz-Dürig-Herzog, Art. 87a, Rdnr. 7; Hernekamp a. a. O., Art. 87a, Rdnr. 4 u. 8; Ipsen, BK, Art. 87a, Rdnr. 17 .

6 BVerfG NJW r 978,1245 .

7 Ebda. S. 1246.

8 Ebda. S. 1247

9 Ebda. S. 1247 unter Verweis auf BVerfGE 28,243 (260).

Io Bt-Drs. 2/2303, S. 31: ^Artikel 4 Absatz 3 befreit somit eine Gruppe von Wehrpflichtigen von einer allgemeinen Staatsbürgerpflicht, er gewährt ein Ausnahmerecht, für das es im deutschen Recht keine Parallele gibt.\& 
Ausgang genommen hatte und sich 1972 in entsprechenden Äußerungen des damaligen Verteidigungsministers Leber fortsetzte. ${ }^{11}$ Einer solchen Verfassungs(um)interpretation hat schon vor 20 Jahren das Bundesverwaltungsgericht die adäquate Antwort erteilt:

„Denn dieses Grundrecht (Art. 4 III GG) wurde schon zu einer Zeit in der Verfassung verankert, in der eine allgemeine Wehrpflicht noch nicht bestand. Es würde widerspruchsvoll sein, wenn man ein in der Verfassung gewährtes Grundrecht als ein Ausnahmerecht gegenüber einer Verpflichtung ansehen wollte, die erst später gesetzlich begründet worden ist. « ${ }^{12}$

Aber auch wenn man mit dem BVerfG Kriegsdienstverweigerer als besondere Gruppe von Webrpflichtigen dem Maßstab des Gleichheitssatzes in Gestalt der Wehrgerechtigkeit unterwirft, stellt der Verzicht auf ein förmliches Prüfungsverfahren keinen Verstoß gegen das Willkürverbot des Art. 3 I GG dar.

Denn selbst das BVerfG kommt angesichts der offenkundigen Schwierigkeiten, Gewissensentscheidungen einer juristischen Prüfung zu unterziehen und der aus Existenz und Ausgestaltung des bisherigen Anerkennungsverfahrens resultierenden tragischen Folgen in Form mehrerer tausend Straf- und Disziplinarverfahren wegen Fahnenflucht und Gehorsamsverweigerung, Flucht von Kriegsdienstgegnern ins Ausland und psychischen Erkrankungen bis hin zum Suizid' 3 nicht umhin, dem Gesetzgeber zu bescheinigen, er habe sich "nicht ohne Grund " zur Reform des fragwürdigen Anerkennungsverfahrens veranlaßt gesehen.

Dieses Zugeständnis wird jedoch im nächsten Atemzug wieder zurückgenommen, wenn gegenüber der im Lichte der Art. I, 3, 20 GG höchst bedenklichen, wenn nicht gar verfassungswidrigen ${ }^{14}$ Realität einer gut zwanzigjährigen Prüfungspraxis das Ideal eines prinzipiell geeigneten Anerkennungsverfahrens beschworen wird und dies ausgerechnet unter Hinweis auf die umstrittene ${ }^{15}$ Rechtsprechung des BVerwG, die durch die Verschärfung der Beweislastregeln maßgeblich zur Verkürzung des Grundrechtsschutzes beigetragen hat.

Auch im Hinblick auf die Nichtüberprüfung der Gewissensentscheidung von Abgeordneten und Eidesverweigerern läßt sich die Frage, warum gerade im Falle der Kriegsdienstverweigerung das Grundgesetz ein Verfahren zur Verhütung von Mißbräuchen gebiete, nur noch durch den Rekurs auf die untergeordnete Position von Art. 4 III GG als eines Ausnahmerechts gegenüber dem Wehrdienst als "gemeinschaftsbezogener Pflicht hohen Ranges ${ }^{16}$ beantworten. Spätestens an dieser Stelle, wo die Notwendigkeit eines Prüfungsverfahrens mit der Bedeutung des Wehrdienstes für die Allgemeinheit begründet wird, erschließt sich auch dem oberflächlichen Betrachter, daß der vom Bundesverfassungsgericht als Grundrechtsbeschränkungstopos strapazierte Gleichheitssatz lediglich dazu diente, angeblich verfassungsrelevante militärpolitische Interessen in das Grundrecht der Kriegsdienstverweigerung hineinzulesen.

II Vgl. Spiegel Nr. $36 / 197^{2}$, S. $4^{8-49}$.

12 BVerwGE 7, 242 (250).

I3 Zu Umfang und Begleiterscheinungen der Delinquenz von Kriegsdienstverweigerern vgl. Krölls a. a. O. (Anm. 2) S. 238-24I.

14 Heilmann-Wahsner, Zur Verfassungsmäßigkeit des Anerkennungsverfahrens für Kriegsdienstverweigerer, JZ 1972, 577 ff.; Kögel, Zivildienstpflicht?, München 1972, S. 59 f.; Lange, Grundwehrdienst und Gewissensfreiheit, MDR 1976,89 (91); Krölls a. a. O., S. 208.

is Vgl. v. Zeschwitz, Das Gewissen als Gegenstand des Beweises, JZ 1970, 233 ff.; Berg, Die Gewissensfrage, MDR 1974, 793 ff.; ders., Anmerkung zum Ureil des BVerwG v. 18. 10. 1972 (NJW 1973, 635), NJW 1973, 1093 f.; VG Wiesbaden NJW I973, 263, Urteil v. 23. 10. 1973 (D III E 145/73).

16 BVerfG NJW I978, 1247 . 
Die Kehrseite des vom BVerfG gepflegten instrumentellen Umgangs mit dem Gleichheitssatz offenbart sich dort, wo das Gericht überraschenderweise dem Gesetzgeber prinzipiell freistellt, auf eine förmliche Gewissensprüfung zu verzichten und statt dessen die Selektion zwischen »echten « und »unechten « Kriegsdienstverweigerern durch die Ausgestaltung des Zivildienstes zu bewirken.

Unter Berufung auf den Gleichheitsgrundsatz propagiert das Gericht als »lästige Alternative ${ }^{17}$ eine "Art verschärften Wehrdienst unter zivilen Bedingungen ${ }^{18}{ }^{18}$ Neben der Angleichung an militärische Lebensformen insbesondere in Gestalt der Kasernierung der Zivildienstleistenden wird schwerpunktmäßig eine Einberufungsquote von $100 \%$ und eine drastische Verlängerung des Zivildienstes empfohlen. Schon im Ansatz erscheint es zweifelhaft, ob ein solcher Funktionswandel des Ersatzdienstes zum reinen Gewissensprüfungsersatz durch das Grundgesetz gedeckt ist. In diesem Zusammenhang kann zustimmend eine Entscheidung des BVerfG aus dem Jahre 196 s zitiert werden, wonach die Organisation des Ersatzdienstes nicht so gestaltet werden darf, »daß sie den Kriegsdienstverweigerer abschreckt und damit auf seine Entscheidung, den Wehrdienst zu leisten oder zu verweigern, einen unzulässigen Druck ausübt. «" ${ }^{19}$ Solche Skrupel plagen das Gericht heutzutage nicht mehr. Unmißverständlich wird formuliert, daß gegenüber der dem Ersatzdienst zugewiesenen Abschreckungsaufgabe das Kriterium der Vergleichbarkeit der Rechte und Pflichten im Wehr- und Zivildienst keine Rolle spiele. Die Grenzen der verfassungsmäßig zulässigen Ungleichbehandlung im Namen der Gleichheit dürften jedoch spätestens mit der vom Gericht erteilten Blankovollmacht zur Verlängerung des Zivildienstes auf 24 Monate überschritten sein. ${ }^{20}$ Eine solche Regelung entspräche nur dann Art. I 2a II S. 2 GG, wenn bei der Bestimmung der Höchstdauer des Zivildienstes nicht auf die tatsächliche Inanspruchnahme der Wehrpflichtigen durch Grundwehrdienst (is Monate) und Wehrübungen (z. Z. durchschnittlich unter I Monat), ${ }^{21}$ sondern auf die gesetzlich zulässige Dauer (is +9 Monate) abgestellt werden könnte. Würde man dem Gesetzgeber einen solchen Spielraum zugestehen ${ }^{22}$, so wäre dem Mißbrauch Tür und Tor geöffnet. Ohne daß die zeitliche Belastung von Wehrpflichtigen auch nur um einen einzigen Tag zuzunehmen bräuchte, könnte man unter Umgehung von Art. I 2a II S. 2 GG durch Heraufsetzung der zulässigen Dauer der Wehrübungen den Ersatzdienst fast beliebig verlängern. ${ }^{22 a}$

IV.

Die materielle Verfassungswidrigkeit der Wehrdienstnovelle beruht nach Ansicht des 2. Senats darauf, daß die gegenwärtige Ausgestaltung der Ersatzdienstpflicht den von ihm aufgestellten Anforderungen an eine »lästige Alternative« nicht genüge.

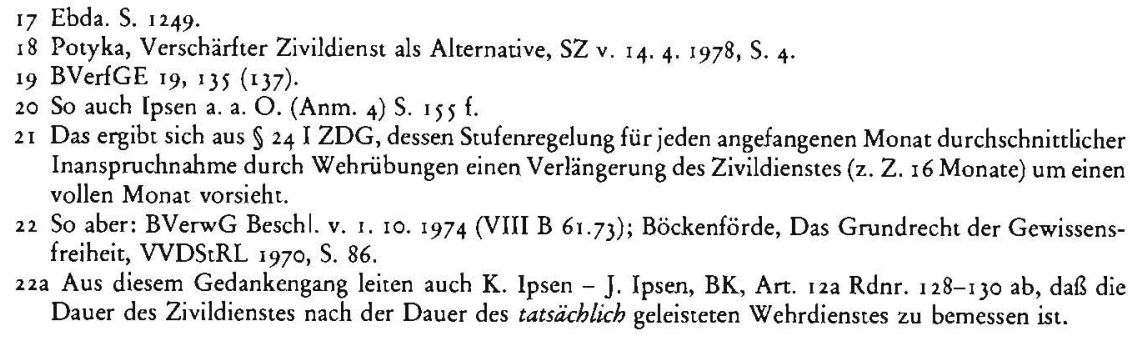


Das Verdikt der Verfassungswidrigkeit reduziert sich in letzter Instanz auf den Vorwurf an die Bundesregierung, sie habe bei ihrer gesetzgeberischen Planung die "gegebene Sachlage" hinsichtlich des Verhältnisses zwischen vorhandenen Zivildienstplätzen und geschätzten Verweigererzahlen verkannt. Mit diesen Erwägungen zur Untauglichkeit einer Gesetzesmaßnahme mit »verfassungsrechtlich unbedenklicher Zielsetzung « ${ }^{23}$ jedoch verläßt der 2. Senat im Widerspruch zu seiner eigenen bisherigen Rechtsprechung den Bereich der verfassungsrichterlichen Normenkontrolle und begibt sich auf das allein dem Gesetzgeber überlassene Feld politischer Zweckmäßigkeitsüberlegungen.

Abgesehen von prinzipiellen Bedenken gegen die Anmaßung gesetzgeberischer Befugnisse läßt sich - wie auch die Gegenrechnung von Hirsch zeigt ${ }^{24}$ - aus dem präsentierten statistischen Material kein so dramatisches Mißverhältnis zwischen der Zahl der Kriegsdienstverweiger und der zur Verfügung stehenden Zivildienstplätze herleiten, wie es das BVerfG tut. Damit soll nicht bestritten werden, daß insbesondere für den Fall einer weiteren Zunahme der Verweigererzahlen in den nächsten Jahren ein Ausbau des Zivildienstes erforderlich gewesen wäre. Um einer möglichen Fehlentwicklung entgegenzuwirken, wäre jedoch ein Auflagenbeschluß zur Erhöhung der Zivildienstkapazitäten das angemessene Mittel gewesen.

\section{V.}

Neben dem fragwürdigen Umgang mit der Statistik findet man wie schon in der Einstweiligen Anordnung ${ }^{25}$ vom Dezember letzten Jahres auch in der jüngsten Entscheidung unbewiesene Behauptungen und Unterstellungen. ${ }^{26}$ Symptomatisch für den Stil des gesamten Judikats ist die von Hirsch zutreffend kritisierte $\mathrm{Art}^{27}$, in der sich der 2. Senat über das entgegenstehende Votum des I. Senates zur Abschaffung des Anerkennungsverfahrens hinwegsetzt. Ebenso unbelegt ist die Aussage, der Wehrdienst sei im Regelfall belastender als der Zivildienst, es sei denn, man sieht den schlichten Hinweis auf fehlende Gemeinschaftsunterkünfte, Uniformierung und laxere Disziplin im Zivildienst als ausreichend an. Hier hätte es zumindest einer ausführlichen Auseinandersetzung mit der detaillierten vergleichenden Darstellung der Rechte und Pflichten von Wehr- und Zivildienstleistenden durch die Bundesregierung ${ }^{28}$ bedurft. Auch hinsichtlich der angestellten Mutmaßungen über einen Zusammenhang zwischen dem Anstieg der Verweigererzahlen und einem behaupteten Motivationswandel ${ }^{29}$ wäre das Gericht gut beraten gewesen, wenn es sich anstelle seines »Augenscheinbeweises « der einschlägigen empirischen Untersuchungen zu Einstellung und Motivation von Kriegsdienstverweigerern ${ }^{30}$ versichert hätte.

23 BVerfG NJW 1978 , I 247 .

24 Ebda. S. 1254 f. Zu einer detaillierten Kritik dieser gegenüber der Einstweiligen Anordnung v. 7. 12. 1977 im wesentlichen unverändert gebliebenen Berechnung vgl. Krölls, Einstweilige Anordnung gegen die Wehrdienstnovelle - Zur Karlsruher Gemeinwohl-Arithmerik, Vorgänge 1978, $20 \mathrm{ff}$.

25 BVerfGE ${ }_{46}, 337=$ NJW 1978, $209=$ BayVBl. 1978, 80 mit Anmerkung Kalkbrenner u. Replik Mainz (BayVBI. 1978, 145).

26 Zur Kritik an den entsprechenden Passagen in der Einstweiligen Anordnung vgl. Kalkbrenner a. a. O., S. $8 \mathrm{if}$

27 BVerfG NJW 1978, $25 \mathrm{r}$.

28 Schriftsatz im Verfassungsstreitverfahren v, 10. I1. 1977, S. 26-29.

29 BVerfG NJW 1978 , I 249

30 Möhle-Rabe, Kriegsdienstverweigerer in der BRD, Opladen 1972; Krölls, Kriegsdienstverweigerung, a. a. O. (Anm. 2); Nagel-Starkulla, Einstellungen von Wehrdienstverweigerern und Soldaten, MünchenMainz 1977. 
Das in diesen Außerungen zu Tage tretende Unbehagen des Gerichts an der Zunahme der Verweigerer in den letzten ro Jahren läßt ein Grundrechtsverständnis erkennen, welches Kriegsdienstverweigerer im Interesse eines angeblichen »Verfassungsgrundsatzes der militärischen Landesverteidigung « auf ein Minoritätendasein zu beschränken sucht. Die Konsequenzen einer solchen mit Verfassungskraft versehenen Auffassung, die das Grundrecht aus Art. 4 III GG zum »Ausnahmerecht für weniger treue Bürger ${ }^{31}$ uminterpretiert, sind von Hirsch aufgezeigt worden. Wird der Vorrang der Gewissensfreiheit zugunsten einer möglichst gerechten Erfüllung einer einfachgesetzlichen Rechtspflicht relativiert, so muß "der Verteidigungsfall folgerichtig zu einer faktischen Außerkraftsetzung von Art. 4 III GG führen. « ${ }^{32}$

Albert Krölls

\section{Staatstreue versus Meinungsfreiheit}

Bemerkungen zur ersten Entscheidung im "Fall Brückner*

Die Bestätigung der vorläufigen Amtsenthebung des Direktors des psychologischen Seminars an der Technischen Universität Hannover, Prof. Dr. Brückner, die der niedersächsische Wissenschaftsminister Eduard Pestel im Oktober 1977 verfügt hatte, durch die Disziplinarkammer beim Verwaltungsgericht Hannover, ist ein gravierendes Alarmzeichen für den Rückgang des staatlichen Respekts vor der Meinungsfreiheit; denn die Sanktionen gegenüber Brückner werden ausschließlich auf Meinungsäußerungen des Wissenschaftlers gestützt, aus denen das Gericht auf seine "feindselige Einstellung«, "feindselige Haltung ", gar "feindliche Gesinnung " gegenüber dem »Rechtsstaat der Bundesrepublik « schließt.' Brückner wird beschuldigt, mit diesen "feindseligen Äußerungen" »die Bundesrepublik, ihre freiheitliche demokratische Grundordnung und ihre verfassungsmäßigen Organe in agitatorischer Form herabgesetzt und damit gegen seine Verfassungstreuepflicht verstoßen" zu haben - ein weiterer, spektakulärer Berufsverbotsfall also, der diese inzwischen zum grauen Alltagsgeschäft der Einschränkung politischer Freiheiten gehörende Praxis um eine weitere Dimension bereichert. Soweit ersichtlich, wird hier zum erstenmal der Versuch unternommen, einen Beamten, der bereits seit Jahren als Lebenszeitbeamter tätig ist, ausschließlich und allein wegen seiner Außerungen - und nicht etwa wegen der Zugehörigkeit zu Parteien oder Organisationen, die von der Exekutive als verfassungsfeindlich eingeschätzt werden, oder wegen sonstiger verfassungswidriger Aktivitäten - »aus dem Dienst zu entfernen «, ein Versuch, mit dessen Erfolg nach Meinung des Gerichts »voraussichtlich ... zu rechnen ist«. Der allseits konstatierte und - wie es den Anschein hat - zunehmend jedenfalls von Sozialdemokraten und Teilen der FDP beklagte Einschüchterungseffekt der Radikalenverfolgung wird sich dann nicht mehr nur auf die Bereitschaft zu engagierter

31 Hirsch, BVerfG NJW 1978, 1252 in Anlehnung an eine Formulierung von Heinemann (NJW 1961, 356 zu BVerfGE I 2, 45).

32 Hirsch, BVerfG NJW 1978, I253.

I Zuerst abgedruckt in: FR v. I1. und I2.9. 1978, Az Dk B 32/77. 